\title{
EXAMINANDO PADRÕES DE DESENVOLVIMENTO TECNOLÓGICO \\ VOLTADO PARA SMART CITY A PARTIR DO DEPÓSITO DE PATENTES
}

\author{
Clarisse Gomes ${ }^{1}$ \\ Gláucia Alves ${ }^{2}$ \\ Gabriel Marcuzzo Do Canto Cavalheiro ${ }^{1}$ \\ ${ }^{1}$ Universidade Federal Fluminense \\ ${ }^{2}$ COPPEAD / Universidade Federal do Rio de Janeiro
}




\title{
EXAMINANDO PADRÕES DE DESENVOLVIMENTO TECNOLÓGICO VOLTADO PARA SMART CITY A PARTIR DO DEPÓSITO DE PATENTES
}

\begin{abstract}
Resumo: O conceito de smart cities tem se consolidado como uma abordagem promissora em resposta aos desafios enfrentados nos centros urbanos. A densidade demográfica nas grandes cidades tende a aumentar, agravando a pressão sob a oferta de transporte, uso da terra e provisão de serviços públicos de qualidade. Tal fato é evidenciado pela evolução crescente no número de iniciativas de smart city e o crescimento exponencial no depóstio de patentes relacionados ao tema. A compreensão das tendências nos projetos de smart city pode apoiar a definição de estratégias e planejamento de iniciativas que promovam maior integração e eficiência das cidades. Este artigo contribui para o entendimento do panorama de smart cities no mundo através da análise do depósito de patentes, evidenciando heterogeneidade na temática dos projetos e baixa relação entre a região de implementação efetiva de soluções e o local de origem do depósito da patente.
\end{abstract}

Palavras-chave: smart city. Cidade inteligente. Patente. Propriedade intelectual.

\section{Introdução}

Nas duas últimas décadas, o conceito de smart city tornou-se cada vez mais popular na literatura científica e nas políticas internacionais (Albino et al, 2015). Em 2008, mais de 50\% da população mundial de 3,3 bilhões de pessoas já vivia em áreas urbanas, e a expectativa é que o percentual chegue a $70 \%$ até 2050 (UN, 2008).

As cidades consomem mais de $60 \%$ da energia global e enfrentam desafios severos para oferta de transporte, uso da terra e provisão de serviços urbanos de alta qualidade (Albino et al, 2015). Neste cenário, a incorporação do conceito de smart city tanto para iniciativas de tecnologia pura quanto na instituição de políticas públicas tem se popularizado com o objetivo de encontrar soluções com efeitos positivos de longo prazo para os desafios urbanos.

Apesar da grande lacuna na provisão de serviços em cidades na América Latina e África, o desenvolvimento de iniciativas de smart city não tem sido homogêneo, se concentrando especialmente nas cidades europeias, norte americanas e na região do Pacífico Ásia (Navigant,

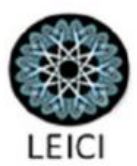


2017). Dentre as áreas endereçadas pelas iniciativas, a maior parte se refere a projetos de "governos inteligentes" relacionados ao atendimento dos cidadãos no que tange a segurança, gerenciamento da população e assistência social. São escassos os projetos que se propõe à utilização mais inteligente da água, ainda que esta seja uma das metas fundamentais para o desenvolvimento urbano, segundo a iniciativa de The Sustainable Development Goals da Organização das Nações Unidas (UN, 2017).

A análise de patentes tem sido indicada para entendimento de fenômenos inovadores (Archibugi and Pianta, 1996) tais como as iniciativas de smart city. Apesar das limitações provenientes da análise de patentes para entendimento da evolução de um mercado, como a não utilização do mecanismo como estratégia de propriedade intelectual, entende-se que esta abordagem seja útil no entendimento dos padrões de dispersão geográfica e temática das iniciativas de smart city.

O entendimento da evolução das iniciativas de smart city e seus impactos sobre os desafios urbanos é de extrema importância para definição das metas para as cidades. A União Europeia já relaciona a implementação de projetos de smart city com o atingimento das metas definidas para 2020 e outros governos tem programas similares de incentivo e encorajamento para desenvolvimento de iniciativas.

Kobayashi et al. (2017) analisou dados patentários relacionados à smart city e constatou uma grande diversidade de soluções tecnológicas, assim como um recente aumento no número de patentes depositadas no European Patent Office (EPO). No entanto, não foi examinada a origem dos pedidos de patentes voltados para smart-city.

Apesar da crescente importância do fenômeno smart city, a literatura carece de estudos voltados para um entendimento sobre a origem das tecnologias voltadas para smart-city. Neste sentido, o objetivo deste artigo é avaliar, através de dados de depósitos de patentes e do mapeamento de iniciativas de smart city, se o padrão geográfico e temático é correspondente e se as patentes têm sido utilizadas como estratégia de propriedade intelectual neste mercado. Verificada a aderência desta hipótese, as tendências em quantidade, localização e temas de iniciativas através do depósito de patentes podem orientar o entendimento da aplicação das iniciativas de smart city em cada região e suas implicações.

\section{Revisão bibliográfica}

\section{SMART CITY}

Não há uma definição amplamente acordada para smart city e uma série de variantes tem sido associada ao termo, como "digital" ou "inteligent" na expectativa de traduzir significados tecnológicos e culturais (Angelidou, 2014, Chourabi, 2012). A popularização deste conceito se fortaleceu nos últimos anos com a pressão sobre os desafios da cidade, 
principalmente sob os efeitos da urbanização, mudanças climáticas, crise financeira e a disputa por trabalhadores qualificados, cidadãos, investidores e turismo (Angelidou, 2014).

A evolução do conceito de smart city parte de um cunho mais tecnológico para uma combinação complexa de tecnologias, fatores sociais e econômicos, arranjos de governança e drivers de políticas e negócios, como pode ser observado na Tabela 1. A implementação do conceito smart city, portanto, segue caminhos muito variados, dependendo das políticas, objetivos, recursos e escopo específicos de cada cidade (Manville et al, 2014).

\section{Tabela 1: Definições de "smart city"}

\begin{tabular}{|c|c|}
\hline Definição & Fonte \\
\hline $\begin{array}{l}\text { O uso de ITC faz os componentes e serviços de infraestrutura crítica de uma cidade - que } \\
\text { incluem administração da cidade, educação, saúde, segurança pública, imobiliário, } \\
\text { transporte e serviços públicos - mais inteligentes, interconectados e eficientes. }\end{array}$ & $\begin{array}{l}\text { Washburn and } \\
\text { Sindhu (2009) }\end{array}$ \\
\hline $\begin{array}{l}\text { As cidades devem ser vistas como sistemas de sistemas e que existem oportunidades } \\
\text { emergentes para introduzir sistemas nervosos digitais, capacidade de resposta inteligente e } \\
\text { otimização em todos os níveis de integração de sistemas. }\end{array}$ & MIT (2013) \\
\hline $\begin{array}{l}\text { Uma cidade "conectando a infra-estrutura física, a infra-estrutura de TI, a infra-estrutura } \\
\text { social e a infra-estrutura de negócios para alavancar a inteligência coletiva da cidade". }\end{array}$ & $\begin{array}{l}\text { Harrison et al } \\
(2010)\end{array}$ \\
\hline $\begin{array}{l}\text { Uma cidade é inteligente quando os investimentos em capital humano e social e infra- } \\
\text { estrutura de comunicação tradicional e moderna alimentam o crescimento econômico } \\
\text { sustentável e uma alta qualidade de vida, com uma gestão sábia dos recursos naturais, } \\
\text { através da governança participativa. }\end{array}$ & $\begin{array}{l}\text { Caragliu, Del Bo } \\
\text { and Nijkamp } \\
(2009)\end{array}$ \\
\hline $\begin{array}{l}\text { Uma cidade inteligente é onde as ICT reforçam a liberdade de expressão e a acessibilidade } \\
\text { a informações e serviços públicos. }\end{array}$ & $\begin{array}{l}\text { Anthopoulos and } \\
\text { Fitsilis (2010) }\end{array}$ \\
\hline $\begin{array}{l}\text { As cidades inteligentes são sobre alavancar a interoperabilidade dentro e entre os domínios } \\
\text { políticos da cidade (por exemplo, transporte, segurança pública, energia, educação, saúde } \\
\text { e desenvolvimento). As estratégias Smart city requerem formas inovadoras de interagir } \\
\text { com as partes interessadas, gerir recursos e prestar serviços. }\end{array}$ & $\begin{array}{l}\text { Nam and Pardo } \\
(2011)\end{array}$ \\
\hline $\begin{array}{l}\text { Uma cidade pode ser chamada de "inteligente" quando os investimentos em capital humano } \\
\text { e social e infra-estrutura de comunicação tradicional e moderna alimentam o crescimento } \\
\text { econômico sustentável e uma alta qualidade de vida, com uma gestão sábia dos recursos } \\
\text { naturais, através da governança participativa. }\end{array}$ & $\begin{array}{l}\text { Schaffers et al. } \\
(2011)\end{array}$ \\
\hline $\begin{array}{l}\text { A Smart city é uma cidade que busca abordar questões públicas através de soluções } \\
\text { baseadas em TIC, com base em uma parceria multipartidária, baseada no município. }\end{array}$ & $\begin{array}{l}\text { European Union, } \\
2014\end{array}$ \\
\hline
\end{tabular}


Ainda que o conceito esteja em construção, Navigant (2017) estima que o mercado global de smart city deve crescer de $\$ 38.8$ bilhões em 2016 para $\$ 83.7$ bilhões em 2025 . Em 2015, foram identificados mais de 250 projetos de smart city em 178 cidades ao redor do mundo, com foco principal em iniciativas de governança e energia, seguido por transportes, construções e recursos hídricos (Navigant, 2017).

Gráfico 1: Projetos de Smart city por tema

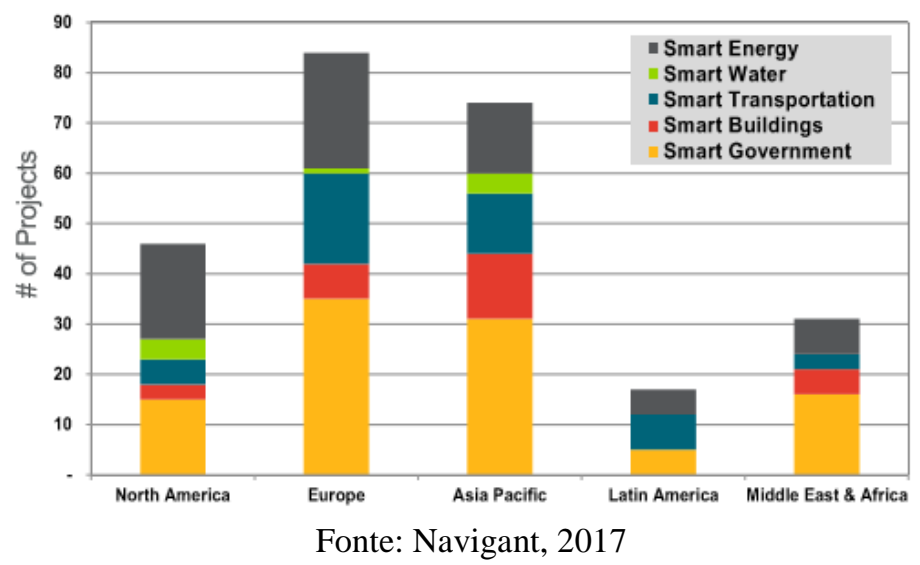

A iniciativas de smart city não são homogeneamente distribuídas, sendo que dois terços das iniciativas mapeadas em smart city estão localizadas na Europa e Ásia. A América Latina apresenta o menor número de iniciativas em desenvolvimento para smart city, não sendo observados projetos relacionado a smart water e smart buildings, apesar dos focos de escassez hídrica e déficit habitacional de qualidade.

As cidades americanas estão cada vez mais focadas na integração de cidades mais inteligentes e diversas ações tem sido feitas na mobilização de stakeholders, tais como o desafio Smart city Challenge, uma iniciativa de departamento nacional de transportes para o desenvolvimento de soluções mais rápidas, baratas e eficientes para mobilidade urbana. As iniciativas se concentram primariamente em energia e governo inteligentes, mas espera-se que o setor de mais rápido crescimento será a água, já que as cidades procuram enfrentar a escassez de água e os problemas de uma infra-estrutura de distribuição em envelhecimento (Navigant, 2017).

A expansão das smart city na Europa são consideradas um veículo útil para que as grandes metas do continente sejam atingidas. Estima-se que 240 cidades europeias tenham planejado ou estão desenvolvendo iniciativas de smart city, sendo Reino Unido, Espanha e Itália os países com maior número de cidades inteligentes (Manville et al, 2014).

Com o seu grande número de megacidades densamente povoadas e economias em rápido crescimento, a região Ásia-Pacífico possui tentativas ambiciosas de direcionar e canalizar tendências de smart city para os objetivos nacionais de desenvolvimento, como o 
imenso corredor industrial Delhi-Mumbai da Índia, os 104 projetos de demonstração da cidade inteligente selecionados na China e o desenvolvimento pioneiro da cidade de Songdo para a Coréia do Sul (Navigant, 2017). Coreia do Sul, Singapura e Japão destacam-se como os países com maior número de iniciativas de smart city na região (Navigant, 2017).

Apesar de enfrentar desafios sociais relevantes, como déficit persistente na moradia de qualidade, insegurança, congestionamento de tráfegos, falta de espaço público e segregação econômica grave, a América Latina registra o menor número de iniciativas identificadas em smart city (Calderon et al, 2017). A literatura é escassa no que tange às iniciativas de smart city na América Latina e o estudo de Calderon et al (2017) conclui que uma política nacional de desenvolvimento tecnológico e articulação de diferentes atores, incluindo o governo, a indústria e as pessoas, é fundamental para implementar soluções inteligentes nestas cidades.

A despeito da ausência de estudos científicos relacionados a smart city na região do Oriente Médio e África, iniciativas são divulgadas principalmente pela Arábia Saudita, onde Dubai emerge como a principal cidade inteligente. Os Emirados Árabes Unidos também são um dos países mais preparados do mundo na segurança cibernética, classificando 17 dos 105 países pela União Internacional das Telecomunicações das Nações Unidas (Khaleej Times, 2016).

Os projetos de smart city são categorizados em seis áreas, segundo definição da União Européia (Manville et al, 2014), o que permite o entendimento da evolução e objetivos das iniciativas planejadas e desenvolvidas. A definição de cada uma das áreas das iniciativas pode ser entendida na Tabela 2.

Tabela 2: categorias de projetos em "smart city"

\begin{tabular}{l|l}
\hline \multicolumn{1}{c|}{ Áreas } & \multicolumn{1}{c}{ Aplicações em smart city } \\
Energia & $\begin{array}{l}\text { Gerenciamento de demanda, suporte de veículo elétrico (EV), programa de eficiência } \\
\text { energética, integração de energia renovável. }\end{array}$ \\
\hline $\begin{array}{l}\text { Água } \\
\text { inteligente }\end{array}$ & $\begin{array}{l}\text { Atualizações do sistema de água, monitoramento do consumo, tratamento de águas } \\
\text { residuais, sistemas de segurança ambiental, gerenciamento de inundações. }\end{array}$ \\
\hline Transporte & $\begin{array}{l}\text { Monitoramento e gerenciamento de tráfego, gerenciamento de congestionamento, } \\
\text { carregamento de usuários rodoviários, compartilhamento de cargas, resposta de } \\
\text { inteligente }\end{array}$ \\
$\begin{array}{l}\text { emergência, sistemas de informação pública, estacionamento inteligente, gerenciamento } \\
\text { integrado de semáforos. }\end{array}$ \\
\hline $\begin{array}{l}\text { Edifícios } \\
\text { Inteligentes }\end{array}$ & $\begin{array}{l}\text { Programas de gerenciamento de energia do setor público, integração de rede para } \\
\text { renováveis, gerenciamento de iluminação / resíduos / água. }\end{array}$ \\
\hline Governo & $\begin{array}{l}\text { Segurança pública, assistência social, tele-saúde, e-educação, dados abertos, iluminação } \\
\text { pública inteligente, portais de cidadãos, gerenciamento inteligente de resíduos. }\end{array}$ \\
\hline
\end{tabular}

Fonte: Manville et al, 2014 
O mapeamento das iniciativas em Smart city realizada por Navigant (2015) demostra assimetria também na distribuição de projetos por tema. Diversos fatores influenciam os caminhos escolhidos pelas cidades para definição das soluções a serem implementadas, sendo as quatro variáveis mais relevantes os fatores estruturais como tamanho e densidade demográfica, desenvolvimento econômico, desenvolvimento tecnológico e políticas ambientalmente favoráveis (Neirotti et al, 2013).

Analisando-se o panorama brasileiro de incentivo para smart city nos últimos anos destacam-se em 2016 o lançamento pela Finep e Fapesp de investimentos de cerca de 10 milhões de reais em projetos relacionados a meio ambiente sustentável, mobilidade urbana, tecnologias assistivas, segurança pública, dentre outros; e o programa Minha cidade inteligente, que busca projetos de uso de rede de fibra óptica e internet das coisas visando a melhoria da gestão das cidades. Em setembro de 2017 o Sebrae anunciou o projeto Cidades Inteligentes, que visa a transformação dos municípios em cidades inteligentes. Além disto, a Capes possui o Programa de Pós-graduação em Cidades Inteligentes Sustentáveis (PPG-CIS) que busca formar mestres com capacidade de desenvolver trabalhos de caráter técnico, científico e de gestão com foco na investigação de possibilidades de promoção da sustentabilidade urbana, por meio de soluções envolvendo sistemas inteligentes em diversas áreas, tendo em vista a melhoria da qualidade de vida. O programa possui três linhas de pesquisas, que caracterizam importantes pilares de estudo em cidades: eficiência energética e recursos naturais, planejamento, dinâmica territorial e mobilidade urbana e sustentabilidade na construção do habitat urbano.

As tendências no desenvolvimento de smart city são uma alavanca para o desenvolvimento urbano global, tendo cada vez mais destaque na definição de políticas públicas. Neste contexto, a identificação e caracterização das iniciativas em desenvolvimento são uma etapa importante para a possibilidade de replicação e consequente escalabilidade das soluções. A análise do padrão de patentes busca entender se esse padrão geográfico e temático é confirmado pelo sistema de propriedade intelectual.

O depósito de patentes de soluções relacionadas a smart city pode trazer, além de um entendimento estratégico de como a propriedade intelectual tem sido tratada neste contexto, evidências sobre a orientação geográfica e temática destas iniciativas ao redor do mundo.

Ao realizar estudo correlacionando smart city e cidades sustentáveis, focado nas bases WoS e Scopus, Kobayashi (2016) concluiu um crescimento significativo no registro de patentes para ambos temas e, especificamente no tema smart city, uma concentração de patentes sobre redes de comunicação sem fio.

\section{ANÁLISE DE PATENTES}

Ao longo das últimas décadas, estudos têm sido desenvolvidos para entender o desenvolvimento tecnológico e seu impacto geográfico e industrial (Archibugi and Pianta, 1996; Basberg, 1987). Neste sentido, algumas correlações têm sido identificadas entre as

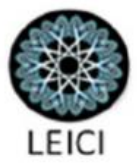


mudanças tecnológicas e os aspectos quanti e qualitativos do depósito de patentes (Daim et al, 2006; Cavalheiro et al., 2014).

Ardito (2017) ressalta que a análise de patentes é a maneira mais adequada para entendimento das tendências de desenvolvimento tecnológico e dinâmicas da inovação. Como o método mais usado para proteção de invenções nas organizações (Archibugi and Pianta, 1996), as patentes consistem em documentos que contém uma "fonte de conhecimento técnico e comercial sobre progresso tecnológico e atividade criativa" (Park et al., 2005).

As desvantagens associadas a análise de patentes residem no fato de que: i) outras estratégias de propriedade intelectual, tal qual o sigilo, podem ser preferidas ao invés das patentes (OECD, 2009), ii) nem todas as invenções podem ser patenteadas (Dernis et al., 2001), iii) a quantidade de patentes não considera necessariamente o valor econômico das invenções (Harhoff et al., 1999). Além disso, considerando a alta conectividade do mundo atual, alguns críticos associam a propriedade intelectual a processos meramente burocráticos (Richard, 2007).

Apesar disso, as patentes trazem vantagens oportunas e tem sido amplamente utilizada sem pesquisas acadêmicas, estratégias de Pesquisa \& Desenvolvimento e estudos de gestão tecnológica (Ardito, 2017). Como apresentado por Pantano (2017), as patentes provem informações detalhadas sore a tecnologia, incluindo atributos técnicos e de Mercado, critérios de originalidade e detalhes sobre o inventor. Patentes também cobrem um campo de inovações ao longo de diferentes países por longos períodos de tempo (Park et al., 2005). Adicionalmente, Cavalheiro et al. (2014) ressalta que o depósito de patentes fornece evidência do nível de interesse comercial por uma determinada área tecnológica.

\section{Hipóteses de pesquisa}

Com base na revisão bibliográfica sobre questões associadas ao uso estratégico da propriedade intelectual por iniciativas de smart city, formulamos três hipóteses de pesquisa que serão investigadas empiricamente neste estudo:

1) H1 - Os países europeus e asiáticos, que detém $2 / 3$ do número de inciativas em smart city, concentram o maior número de depósitos de IP (intelectual properties) em comparação com outras regiões.

2) H2 - Projetos relacionados a governança e energia, mais frequentes em iniciativas de smart city, registram o maior número de depósitos de IP (intelectual properties) em comparação com os demais temas. 
3) H3 - O número de documentos de patente de países líderes em projetos de smart city provavelmente aumentarão ao longo do tempo, à medida que os projetos se tornam mais maduros.

\section{MÉTODO DE PESQUISA}

Tendências em tecnologia têm se beneficiado da análise de patentes através da análise bibliométrica e da extração de textos (Pantano, 2017). Enquanto a primeira busca indicadores baseados em matemática ou estatística, como o número de patentes em determinado período de tempo (Lee et al., 2011), a extração de texto aporta uma visão mais detalhada da descrição dos documentos, ambos através de um processo de busca baseado em palavras chave (Madani, 2016).

A pesquisa e análise do depósito de patentes foi realizada a partir do repositório global de patentes do European Patent Office. O critério de pesquisa utilizado foi composto pelas interseções entre as palavras chave "SMART CITY"; "SMART ENERGY", "SMART WATER", "SMART TRANSPORTATION", "SMART BUILDINGS" e "SMART GOVERNMENT". A pesquisa às patentes limitou-se às concessões entre 2012 e 2016, dado a emergência do tema a partir deste ano (Roland Berger, 2017).

Esse procedimento permitiu coletar 483 patentes. Para refinamento dos resultados, foi feita uma triagem com a intenção de eliminar inscrições que não se referiam a iniciativas de smat city. $\mathrm{O}$ banco de dados foi organizado por número de aplicação, título, resumo, data do pedido, data de aceitação, cessionários e país. Cada patente foi classificada, pelo grau de proximidade, a cada um dos temas de definição de smart city, conforme definições apresentadas na Tabela 2.

Finalmente, foi analisada a frequência de patentes por país e por área de definição, correlacionando estes resultados com o número total de projetos relacionados a smart city apresentado em Navigants (2017).

\section{RESULTADOS}

A pesquisa de patentes entre 2012 e 2016 relacionadas a smart city retornou 483 depósitos e concentrados em apenas 12 países. Através da análise dos resumos, descrições e reclamações foram classificados em cada uma das 6 áreas temáticas, sendo que 128 dos registros não puderam ser classificados. 
Gráfico 2: Registros de patentes de smart city por país entre 2012 e 2016

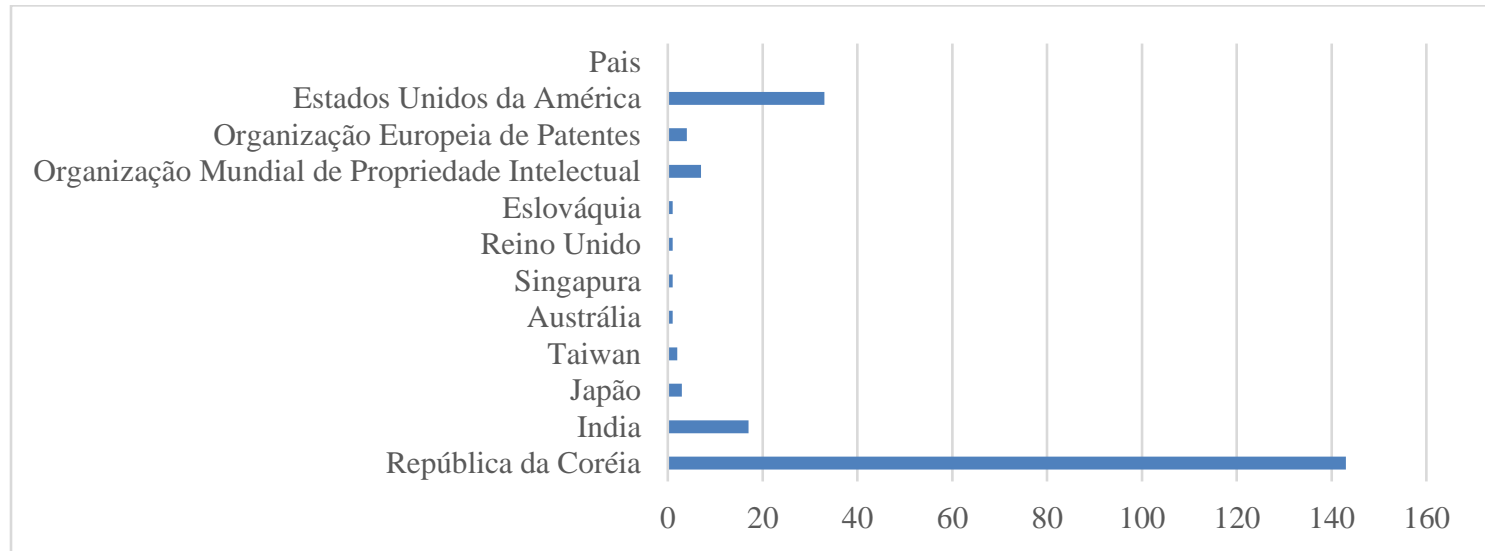

Fonte: Autores

Majoritariamente, o depósito de patentes de projetos de iniciativas de smart city se concentra na região Pacífico Asiático. Não foram observados depósitos de patentes nas regiões da América Latina, África e Oriente Médio.

\section{Gráfico 3: Distribuição de patentes por região e tema}

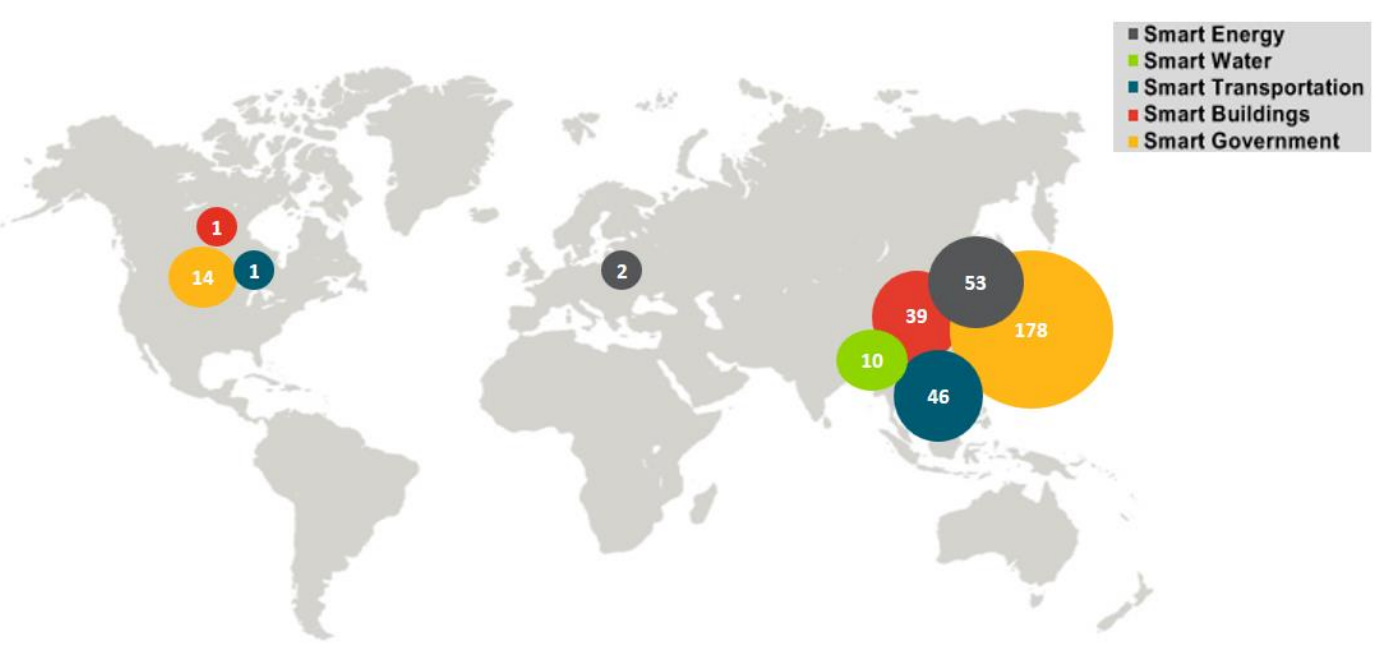

Fonte: Autores

Mais de $40 \%$ dos depósitos de patentes relacionados a smart city se referem a governos inteligentes, sendo a maioria tanto dos pedidos de patentes norte americanos como na região Pacífico Asiático. Soluções de água inteligente foi o tema com menor número de citações nos depósitos de patentes. 
O número de depósitos de patentes em smart city tem seguido uma curva exponencial desde 2012, onde partiu de 13 registros alcançando 296 registros em 2016.

\section{Gráfico 4: Evolução geral do registro de patentes em smart city}

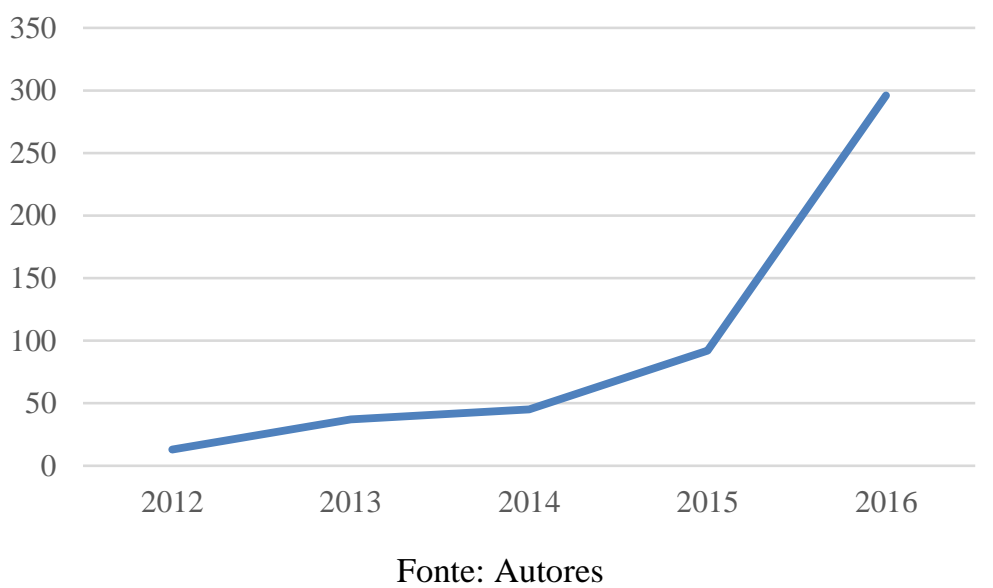

Considerando a evolução dos depósitos por país, exceto pelo dado da República da Coréia em 2012, os números têm crescido substancialmente especialmente na China, índia, República da Coreia e Estados Unidos.

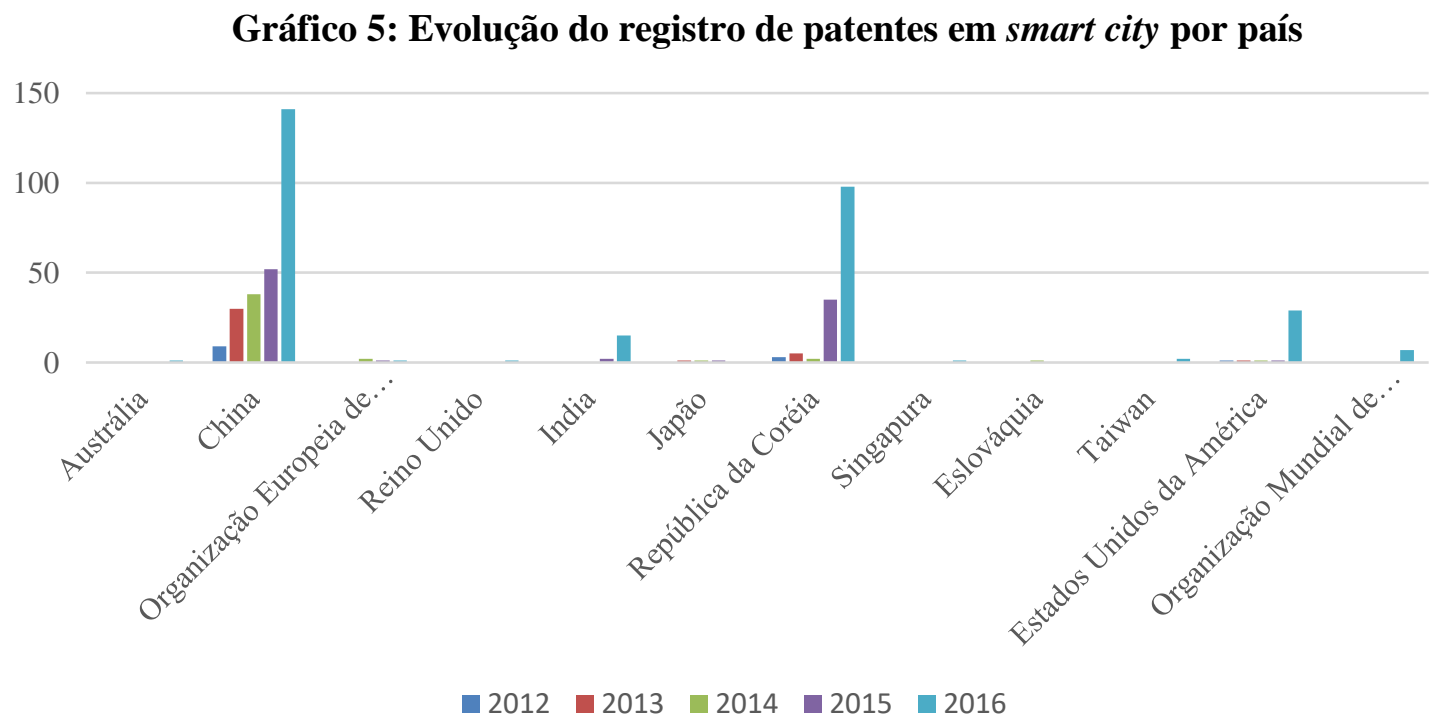

Fonte: Autores

Muitos países fizeram seu primeiro depósito em 2016, como reino Unido, Singapura e Austrália, o que reforça a contemporaneidade do tema no contex to mundial. 
Quando comparado o número de iniciativas mapeadas por Navigant (2017) com a quantidade total de depósitos de patentes entre 2012 e 2016 verifica-se uma correlação baixa entre as duas fontes.

Na região da América do Norte a correlação foi mais próxima, sendo identificados 46 iniciativas de smart city e 33 patentes depositadas. A maior discrepância foi observada na Pacífico Ásia, onde foram identificados 437 depósitos de patentes mas apenas 74 iniciativas foram contabilizadas. Na Europa foram depositadas apenas 2 patentes, apesar dos 84 projetos mapeados.

As regiões da América Latina e Oriente Médio e África não apresentaram depósito de patentes e tem número pouco expressivo de iniciativas de smart city mapeadas.

Gráfico 6: relação geral entre projetos mapeados e patentes registradas em smart city

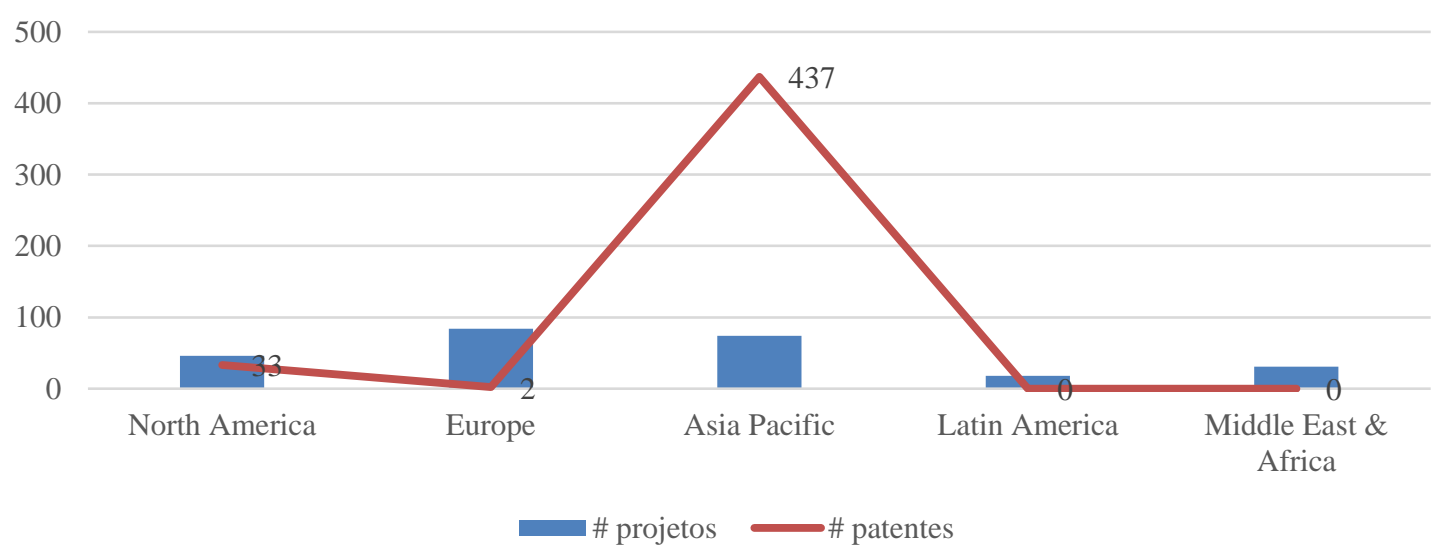

Fonte: Autores

Quando observada a distribuição de patentes por tema, observa-se que a discrepância entre o número de projetos e o de patentes na região da Ásia Pacífico se dá principalmente pelos depósitos em Smart energy. São 178 patentes depositadas neste tema e 13 projetos identificados. Na Europa a correlação entre temas foi próxima com exceção de Smart energy, que teve 19 projetos identificados e nenhuma patente depositada. 
Gráfico 7: relação projetos mapeados e patentes registradas em smart city por região

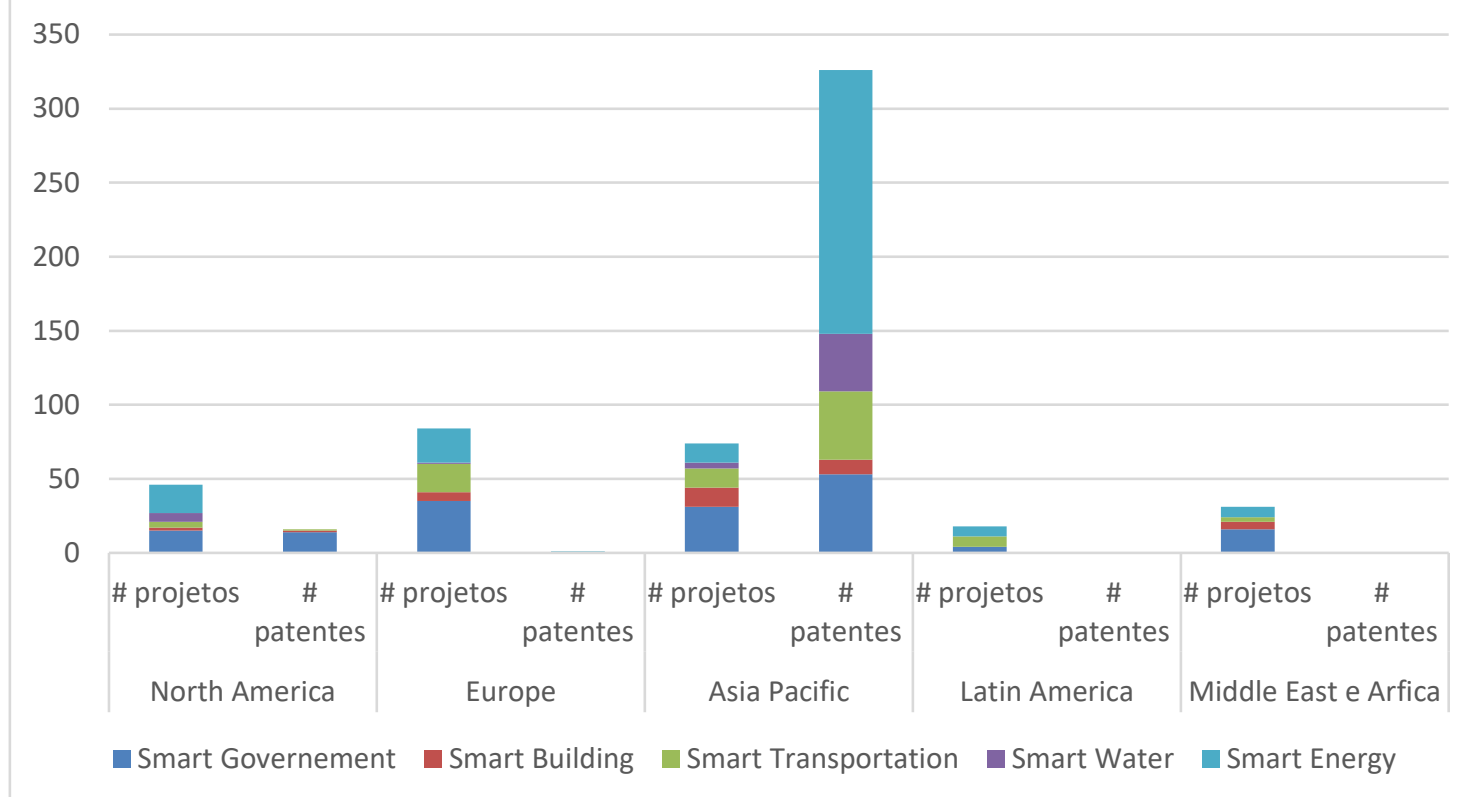

Fonte: Autores

\section{DISCUSSÃO}

Com base no levantamento e análise do depósito das patentes, em relação às hipóteses levantadas no presente estudo, é possível perceber que a primeira hipótese trazia a correlação entre o número de iniciativas e o de IP para os países asiáticos e europeus. Interessante observar que o continente asiático possui esta correlação como verdadeira, uma vez que possui elevado número de iniciativas e elevado número de depósitos de IP, superando inclusive a correlação de proporção com as demais regiões geográficas em relação às iniciativas. Já para a Europa, apesar de volume significativo de iniciativas, o índice de depósitos de IP é baixo. Muitas possibilidades podem ser levantadas a partir desta constatação, como o fato de depósitos de IP poderem representar um maior potencial de aplicação de iniciativas futuras no continente asiático ou de a Europa já possuir um caráter de desenvolvimento de smart city focado na utilização de práticas que já possuem depósito de IP de outras fontes, sendo assim mais um reutilizador de soluções do que propriamente inovador. Também há a possibilidade da aplicação dos projetos na Europa, mesmo que inovadores, estar sendo realizada sem o endereçamento de IP. Outra possibilidade é de os depósitos de IP relativos a este continente serem realizados sob nomenclatura diferente de smart city, não sendo assim contemplados nos dados analisados no presente estudo. Esta hipótese é, portanto, parcialmente verdadeira.

Quanto à segunda hipótese aqui levantada, os projetos relacionados a governança e energia, mais frequentes em iniciativas de smart city, registram sim o maior número de registros de IP em comparação com iniciativas de transportes, construções e recursos hídricos, 
demonstrando assim uma correlação entre os registros de IP e os projetos realizados. Se esta correlação se manter verdadeira, estudos sobre o progresso de registros de IP em demais temáticas de smart city poderão indicar tendências de desenvolvimento de projetos futuros. Neste contexto, existirá uma significativa tendência de desenvolvimento de smart energy no continente asiático, por exemplo.

Quanto à terceira e última hipótese do presente estudo, interessante observar que a discrepância entre projetos, iniciativas e IP observada na primeira hipótese demonstra que não há relação direta entre o número de documentos de patente de países líderes em projetos de smart city e o de iniciativas e projetos, não sendo possível afirmar que o registro de IP aumentará ao longo do tempo, à medida que os projetos se tornam mais maduros. Vide exemplo claro do continente europeu, onde o número de projetos é significativamente maior que o número de registro de IP.

\section{CONCLUSÃO}

Os resultados apresentados neste estudo demonstram um crescimento acentuado no depósito de patentes relacionados a smart city nos últimos 4 anos. Não foi observada uma homogeneidade com relação à temática e geografia, já que a maior parte dos registros se localizam na região Ásia Pacifico relacionados a soluções de Smart Governement.

Com relação à localização, os continentes americano e europeu além da região Ásia Pacífico registram o maior número de projetos em andamento em Smart city e também maior volume de depósito de patentes relacionado ao tema.

Smart Governement e smart energy são os temas mais recorrentes tanto para iniciativas de smart city quanto para registro de patentes. Tal fato aponta para maior probabilidade de que evoluções sejam percebidas no que tange a otimização da jornada dos cidadãos e ao ciclo da energia nas cidades.

A fraca relação entre as regiões onde iniciativas de smart city estão sendo implementadas e os pedidos de patentes são realizados podem indicar uma migração tecnológica entre regiões ou que diferentes estratégias de inovação são consideradas neste campo. Estudos específicos em cada região com relação à estratégia de propriedade intelectual dos projetos de smart city podem esclarecer este movimento.

Estudos mais detalhados relacionados ao continente europeu visando entender a discrepância entre o volume significativo de iniciativas e projetos e o baixo de registro de IP sob o nome smart city podem ser interessantes na previsão de tendências de desenvolvimento neste continente.

Outro campo interessante a ser pesquisado são as motivações para o elevado registro de IP do continente asiático em relação ao tema smart city, podendo demonstrar incentivos 
governamentais relevantes, tendências econômicas e de desenvolvimento. Para esta pesquisa sugere-se entrevistas com pesquisadores de empresas locais para entendimento dos motivos de registro e dos planos de futura aplicação das patentes em projetos.

\section{REFERÊNCIAS BIBLIOGRÁFICAS}

Albino, V., Berardi, U. and Dangelico, R.. (2015). "Smart city: Definitions, Dimensions, Performance, and Initiatives." Journal of Urban Technology, Vol 22, No 1, pp. 3-21

Angelidou, M. (2014). "Smart city: a conjucture of four forces". City, Vol 47, pp. 95-106

Ardito, L., D'Addab, D., Petruzzellia, A. (2017). "Mapping innovation dynamics in the Internet of Things domain: Evidence from patent analysis". Technological Forecasting \& Social Change.

Archibug, D. and Pianta, M. (1996). "Measuring technological through patents and innovation surveys". Technovation 16 (9), 451-468.

Basberg, B. (1987). "Patents and the measurement of technological change: a survey of the literature”. Res. Policy 16 (2), 131-141

Bell, M. (2006), "Time and technological learning in industrialising countries: how long does it take? How fast is it moving (if at all)?" International Journal of Technology Management, Vol. 36 Nos 1/2/3, pp. 25-39.

Calderón, M., López, G. and Marín, G. (2017). "Smart city in Latin America Realities and Technical Readiness". Springer International Publishing, pp. 15-26.

Cavalheiro, G., Joia, L.A., and Gonçalves, A.C. (2014). "Strategic patenting in the upstream oil and gas industry: assessing the impact of the pre-salt discovery on patent applications in Brazil". World Patent Information, 39, pp. 58-68.

Chourabi, H., Taewoo, N., Walker, S., Garcia, R., Mellouli, S., Nahon, K., Pardo, T. and Scholl, H. (2012). "Understanding smart city: An integrative framework" In System Science (HICSS), 2012 45th Hawaii International Conference on, pp. 2289-2297.

Daim, T., Rueda, G., Martin, H., Gersdri, P. (2006). "Forecasting emerging technologies: use of bibliometrics and patent analysis". Technol. Forecast. Soc. Change 73, 981-1012.

Dernis, H., Guellec, D., Bruno van Pottelsberghe de la, P. (2001). Using patent counts for crosscountry comparisons of technology output. STI Rev. 27, 129-146.

Harhoff, D., Narin, F., Scherer, F.M., Vopel, K. (1999). "Citation frequency and the value of patented inventions". Rev. Econ. Stat. 81, 511-515.

Khaleej Time, 2016. "Dubai tops Middle East's smart city list". Available at https://www.khaleejtimes.com/technology/dubai-tops-middle-easts-smart-city-list. Access in Nov 2017. 
Kobayashi, Andrea Regina Kaneko et al. Smart sustainable cities: bibliometric study and patent information. International Journal of Innovation (iji), [S.1.], v. 5, n. 1, p. 77-96, feb. 2017. ISSN 2318-9975. Disponível em: 〈http://www.journaliji.org/index.php/iji/article/view/159>. Acesso em Nov 2017.

Lee, C., Jeon, J., Park, Y. (2011). "Monitoring trends of technological changes based on the dynamic patent lattice: a modified formal concepts analysis approach". Technol. Forecast. Soc. Change 78 (4), 690-702

Manville, C., Cochrane, G., Cave, G., Millard, J., Pederson, Thaarup, R., Liebe, A., Wissner, R., Massink, R., Kotterink, B. (2014). "Mapping Smart city in the EU”. European Parliament. Navigant Research Consulting (2017). "Smart city: trends, opportunities, and challenges".

Neirotti, P., Marco, A., Cagliano, A., Mangano, G., Scorrano, F. "Current trends in Smart city initiatives: Some stylised facts". Cities, Vol, pp. 25-36

OECD, 2009. OECD Patent Statistics Manual. OECD Publishing.

Pantano, E., Priporas, C., Sorace, S., Iazzolino, G. (2017). "Does innovation-orientation lead to retail industry growth? Empirical evidence from patent analysis". Journal of Retailing and Consumer Services, Vol 34, pp. 88-94

Park, Y., Yoon, B., Lee, S. (2005). "The idiosyncrasy and dynamism of technological innovation across industries: patent citation analysis". Technol. Soc. 27 (4), 471-485.

Richard, N. (2007). The changing institutional requirements for technological and economic catch up. International Journal of Technological Learning, Innovation and Development (IJTLID), Vol. 1, No. 1

Roland Berger. (2017). "Smart city, smart strategy". Roland Berger GMBH

UN, United Nations. (2008). "World Urbanization Prospects: The 2007 Revision Population Database". Available at http://esa.un.org/unup/ 